\title{
Differentiation of Bone Marrow Mesenchymal Stem Cells into Alveolar Epithelial Cells Reduces Radiation-Induced Lung Injury by Regulating Angii/ACE2/Ang(1-7) Axis and Suppressing NF- Kb/MAPK Pathway
}

shiying niu

Shandong Academy of Medical Sciences

changsheng cong

Jinan Central Hospital

zhaopeng wang

Shandong First Medical University

meili sun

Jinan Central Hospital

yueying zhang ( $\sim$ zhangyueying828@163.com )

The First Affiliated Hospital of Shandong First Medical University https://orcid.org/0000-0002-50062483

\section{Research}

Keywords: Radiation-induced lung injury, Bone mesenchymal stem cells, Renin-angiotensin system, MAPK, NF-KB

Posted Date: September 20th, 2021

DOI: https://doi.org/10.21203/rs.3.rs-882694/v1

License: (c) (i) This work is licensed under a Creative Commons Attribution 4.0 International License.

Read Full License 


\section{Abstract}

Background Radiation-induced lung injury (RILI) is one of the most common complications of thoracic tumors radiotherapy. Since therapeutic strategies remains limited, the exploration of new approaches to treat RILI is on high demands. The use of bone mesenchymal stem cells (BMSCs) to treat RILI holds great promise thanks to their multidifferentiation and anti-inflammatory potential after injury. Here, we investigate the therapeutic potential of BMSCs in RILI.

Methods Forty five C57BL/ 6 mice were randomly divided into groups. Except for the control group, all mice received chest irradiation. Within 24 hours after irradiation, BMSCs were injected into the tail vein of mice in BMSCs group. At 4 weeks after irradiation, all mice were dissected. HE staining and immunohistochemistry were used to observe the pathological changes of lung tissue and the expression of inflammatory factors. Immunofluorescence technique was used to detect whether BMSCs migrated to lung tissue and to verify their differentiation potential. The expression of Ang II and Ang (1-7) in lung tissue was detected by ELISA. The expression of MasR mRNA in lung tissue was detected by qRT-PCR. Western blotting was used to detect the expression of ACE2, ACE, AT1R and MAPK related proteins.

Results we found that BMSCs significantly reduced RILI by HE and immunohistochemistry. Immunofluorescence results showed that BMSCs migrated to injuried lung tissue and differentiated into alveolar epithelial cells. Combined with qRT-PCR and Western blotting results showed BMSCs significantly up-regulated ACE2/Ang(1-7)/MasR axis and suppressed NF-KB/MAPK pathway.

Conclusions The study demonstrated that BMSCs may be transplanted into damaged lung tissue where they differentiated into AEC II to regulate Angll/ACE2/Ang(1-7) axis and suppress NF-KB/MAPK pathway to alleviate RILI.

\section{Introduction}

Over the last few decades, radiation therapy (RT) has been remained a cornerstone of treatment whether radical or palliative treatment for many malignancies [1]. Although technology improvements in radiotherapy, The occurrence of radiation-induced lung injury (RILI) is still inevitable [7]. It is mainly divided into two stages-radiation pneumonitis (RP) and radiation-induced pulmonary fibrosis (RIPF), which represents irreversible damage [8]. Due to the precise mechanism of RILI is not fully clear, there is still a lack of effective treatment [1]. Bone mesenchymal stem cells (BMSCs), a pluripotent stem cell, have the positivel effect for regenerative medicine [9]. It has been reported that BMSCs can migrate to sites of tissue injury and release anti-apoptotic, anti-inflammatory and angiogenic factors to exert a series of effect [10]. Studies have showed that BMSCs may exert great therapeutic potential in several diseases, including acute lung injury (ALI), acute respiratory distress syndrome (ARDS) [1]. Therefore, these studies suggested that BMSCs might be a potential therapy in RILI.

RAS plays a significant role in modulating blood pressure homeostasis, as well as fluid and salt balance[11]. Whereas, there is a growing body of evidence suggested that it was also important in 
regulating inflammatory responses [4]. ACE2, a homologue of ACE and a negative regulator of the reninangiotensin system (RAS), downregulated the protein expression of angiotensin II through catalyzing the conversion of Ang II to angiotensin-(1-7) (Ang-(1-7)), which restrains the vasoconstrictive and inflammatory reaction [12]. Besides, research showed recombinant ACE2 significantly reduced the expression levels of inflammatory cytokines and protected from severe acute lung failure [13]. The RILI may occur due to a series of inflammatory reactions caused by cell damage. Alveolar epithelial cells, which are sensitive to irradiation, were the first to be damaged. Due to cell injury, the expression of ACE2 was decreased, which aggravated RILI. MSCs have potential as regenerative therapeutics due to differentiation potential, which are able to repair damage in a number of tissues. However, detailed mechanism of this effect and the downstream molecular have not yet been fully elucidated.

Mitogen-activated protein kinases (MAPKs) signaling pathway plays an significant role in immune and inflammatory responses[14]. It is consisted of stress-activated JNK, p38 and growth factor-regulated ERK1/2. NF-KB pathway can be activated by MAPKs and modulated transcription of inflammatory factors $[15,16]$. Previous studies have reported that up-regulation of the ACE2/Ang-(1-7)/Mas axis protected against sepsis-induced acute lung injury by inhibiting the MAPK/NF-kB pathway [6].

Therefore, our study mainly discussed the mechanism of BMSCs protects against RILI. We showed that BMSCs could differentiate into alveolar epithelial cells to regulate ACE2/Ang(1-7)/Mas axis and inhibit NF-KB/MAPK signaling pathway to protect from RILI.

\section{Methods}

\section{Isolation and culture of BMSCs}

The BMSCs are a cell line obtained from rats thighbone. First, we took out the femur of rats with intact femoral head, washed the femur with $75 \%$ alcohol and double antibody, then removed the epiphyseal end and exposed the bone marrow cavity, finally the culture medium was used to wash the cells. BMSCs cells were cultured with OriCell Wistar Rat Bone Marrow Mesenchymal Stem Cell Medium supplemented with 10\% OriCell Superior-Qualified Fetal Bovine Serum, Penicillin-Streptomycin and L-Glutamine (Cyagen Biosciences, China) at $37^{\circ} \mathrm{C}$ in a humidified atmosphere of $5 \% \mathrm{CO}$. Non-adherent cells were removed by changing the culture medium after incubation for 24 hours. The medium was replaced every 2-3 days. All the experiments in this study were carried out using 4 th generation cells.

\section{Identification of BMSCs}

To identify whether they are BMSCs, Immunofluorescence was used to detect expression of CD34 and CD44 of BMSCs. The sections containing BMSCs were fixed with $4 \%$ paraformaldehyde for 30 min and blocked by $5 \%$ goat serum at $37^{\circ} \mathrm{C}$ for $30 \mathrm{~min}$. BMSCs were incubated with antibody CD34 and antibody $\mathrm{CD} 44$ at $4^{\circ} \mathrm{C}$ overnight. Then BMSCs were incubation with Alexa Fluor 594 conjugated goat anti-rabbit IgG antibodies (1:50, \#ZF-0516, ZSGB-BIO, Beijing, China) for visualization. The nuclei were stained with 
4,6-diamidino-2-phenylindole (DAPI). The fluorescent signal was showed using 3D scanner (3DHISTECH, Hungary).

To test the multidifferentiation potential of BMSCs toward the osteogenic and adipogenic, the BMSCs were cultured in adipogenic and osteogenic differentiation medium (Cyagen Biosciences, China). After 4 weeks of differentiation induction, adipose cells were stained with Oil Red $\mathrm{O}$ and osteogenic cells were were stained with Alizarin Red. The stained cells were observated by a microscope.

\section{Animals and Experimental models}

45 male C57BL/ 6 mice with an average body weight of $20 \mathrm{~g}$ at 8 weeks of age purchased from Jinan pengyue experimental animal co., Ltd were utilized for all experiments. All animal procedures were approved by the Ethics Committee of The First Affiliated Hospital of Shandong First Medical University. Totally 45 Mice were evenly divided into three groups including control group, model group and BMSCs group with 15 mice in each group. Thorax irradiation was delivered to all except the mice from control group. The mice were anesthetized by intraperitoneal injection of $10 \%$ chloral hydrate $(0.04 \mathrm{ml} / 10 \mathrm{~g})$ and exposed to chest irradiation at a dose rate of $2 \mathrm{~Gy} / \mathrm{min}$, with a total dose of $20 \mathrm{~Gy}$. Within 24 hours after irradiation, the mice in BMSCs group were injected with BrdU labeled BMSCs $\left(1 \times 10^{6}\right.$ cells $\left./ 0.1 \mathrm{ml}\right)$ via tail vein. All the mice were sacrificed 4 weeks after irradiation. The lung tissues were procured for histological and molecular biological analysis.

\section{Animal sample collection}

Four weeks after irradiation, the mice were anesthetized by intraperitoneal injection of $10 \%$ chloral hydrate $(0.04 \mathrm{ml} / 10 \mathrm{~g})$. Firstly, the eyeballs of the mice were removed and the blood was collected. The supernatant was collected by centrifugation and frozen at $-20^{\circ} \mathrm{C}$. The left lung was cryopreserved at $80{ }^{\circ} \mathrm{C}$ to prepare for the molecular experiment. The right lung was fixed in $10 \%$ formaldehyde for pathological experiment.

\section{Histologic examination}

H\&E staining was performed to evaluate the pathological changes of lung tissue. Briefly, the lung tissues were fixed in 10\% formaldehyde, paraffin-embedded and then sectioned into $4 \mu \mathrm{m}$ slices (Leica, Germany). Then the nucleus and cytoplasm were stained with hematoxylin and eosin respectively, the neutral gum was used for sealing. All sections were observed under light microscope. Immunohistochemistry staining was used to detect expression of inflammatory factors in lung tissues. The paraffin-embedded lung tissue sections were dewaxed to water, repaired by EDTA for $15 \mathrm{~min}$ at $100^{\circ} \mathrm{C}$, then incubated with $5 \% \mathrm{BSA}$ (Solarbio, Beijing, China) for $30 \mathrm{~min}$ at $37^{\circ} \mathrm{C}$. The slides were incubated with Interleukin 6 (IL-6) (1:100, \#bs-0782R, Bioss, Beijing, China) antibody and Tumor necrosis 
factor a (TNF-a) (1;100, \#bs-10802R, Bioss, Beijing, China) at $4{ }^{\circ} \mathrm{C}$ overnight. Subsequently, the slides were incubated with secondary antibody (1:200, \#bs-0295D-HRP, Bioss, Beijing, China) for 60 min at $37^{\circ} \mathrm{C}$. The slides were visualized by 3,3-diaminobenzidin (DAB). Finally, the slices were stained with hematoxylin, dehydrated with gradient alcohol, transparent with xylene and sealed with neutral gum. The positive signal was determined by a 3D scanner (3DHISTECH, Hungary).

\section{Cells and tissues Immunofluorescence}

The BrdU-Labeled BMSCs and lung tissue sections were fixed with $4 \%$ neutral formaldehyde solution for $15 \mathrm{~min}$, then denatured with $2 \mathrm{~m}$ hydrochloric acid for 15 minutes, neutralized with sodium borate and washed with distilled water, followed with $0.1 \%$ Triton X-100 for 10 min and incubated with $5 \%$ BSA (Solarbio, Beijing, China) for $30 \mathrm{~min}$ at $37^{\circ} \mathrm{C}$. The cells were then incubated with primary antibodies: mouse anti-BrdU antibody (1:100, \#ZM-0013, ZSGB-BIO, Beijing, China) and rabbit anti-AQP5 (1:100; \#bs10927R, Bioss, Beijing, China) and rabbit anti-Pro-spc (1:200; \#ab90716, Abcam, China) at $4{ }^{\circ} \mathrm{C}$ overnight. Subsequently, these cells were incubated with the corresponding goat anti-mouse $\lg G(\mathrm{H}+\mathrm{L})$ secondary antibody, Alexa Fluor 488 ((1:50, \#ZF-0512, ZSGB-BIO, Beijing, China) and goat anti-rabbit $\operatorname{lgG}(\mathrm{H}+\mathrm{L})$ secondary antibody, Alexa Fluor 594 ((1:50, \#ZF-0516, ZSGB-BIO, Beijing, China) for 60 min at $37^{\circ} \mathrm{C}$. The cell nuclei were counterstained by DAPI. The cells stained with green fluorescence were counted as the BrdU-positive cell. The cells stained with red fluorescence were counted as the AQP5 and Pro-spc positive cell. BrdU positive and AQP5 positive or Pro-spc positive were positive signal. Immunofluorescence images were detected by a 3D scanner (3DHISTECH, Hungary).

\section{Enzyme-linked immunosorbent assay (ELISA)}

We used a commercially available mouse ELISA kits, according to the manufacturer's instructions, to detect the levels of Angll (CK-E21284, MLBIO, Shanghai, China) and Ang-(1-7) (CK-E20733, MLBIO, Shanghai, China) in lung tissues. The absorbance was read at $450 \mathrm{~nm}$ with a microplate reader.

\section{qRT-PCR}

Total RNA was extracted from freshly isolated lung tissues using Trizol reagent (Vazyme biotech co., Nanjing, China) according to the manufacturer's instructions. Then RNA was reverse-transcribed into cDNA with cDNA Synthesis Kit (Vazyme Biotech Co., Nanjing, China). Quantitative real-time PCR was performed with SYBR qPCR Mix (Vazyme Biotech Co., Nanjing, China). The mRNA levels of MasR in lung tissues were examined by qRT-PCR. Data were calculated using the $2^{-\triangle} \triangle \mathrm{t}$ method. MasR forward primer 5'-ACAACACGGGCCTCCTATCTG-3' and reverse primer 5'-GAAGGGCACAGACGAATGCT-3',

$\beta$-actin forward primer 5'-GGCTGTATTCCCCTCCATCG-3' and reverse primer 5'-

CCAGTTGGTAACAATGCCATGT-3'. 


\section{Western blots}

Western blots was used to analyse the related proteins of ACE/ACE2/ Ang(1-7) and MAPK parhway in lung tissue. The lung tissues were lysed in RIPA lysis buffer (Beyotime, China) with protease inhibitors for $30 \mathrm{~min}$ at $4^{\circ} \mathrm{C}$, followed by centrifugation at $12,000 \mathrm{rpm}$ for $15 \mathrm{~min}$ at $4{ }^{\circ} \mathrm{C}$. The supernatant will be collected and added to the loading buffer for denaturation. Protein samples were separated by $8-15 \%$ SDS-PAGE, then transferred to a PVDF membrane and blocked in 5\% skim milk at room temperature for 90 min. The membranes were incubated with primary antibody including ACE (1:1000; \#bs-0439R, Bioss, Beijing, China), ACE2 (1:1000, \#21115-1-Ig, Proteintech, China ), AT1R (1:1000; \#bs-0630R, Bioss, Beijing, China), p38 (1:1000囚\#OM125772, OmnimAbs, China), p-p38(1:1000; \#bs-0636R, Bioss, Beijing, China), Jnk (1:1000冈\#OM260186, OmnimAbs, China), p-Jnk (1:1000; \#bs-17591R, Bioss, Beijing, China), ERK1/2 (1:1000囚\#OM125780, OmnimAbs, China), p-ERK1/2 (1:1000; \#bs-3016R, Bioss, Beijing, China), NF-KBp65 (1:1000, 66535-1-Ig, Proteintech, China), NF-KBp-p65 (1:1000; \#bs-3485R, Bioss, Beijing, China),overnight at $4{ }^{\circ} \mathrm{C}$ and the corresponding secondary antibody at room temperature for $1 \mathrm{~h}$. Finally, proteins were visualized with ECL(Milipore, USA) and analyzed with Image J.

\section{Statistical analysis}

All data are shown as mean \pm SEM. Data was analyzed using SPSS 25 software. The measurements for this study were performed with one-way analysis of variance (ANOVA) for multiple groups. $P<0.05$ was considered statistically significant.

\section{Results}

\section{Assessment of BMSCs}

The BMSCs are a cell line obtained from rats thighbone. During the process of cell expansion, fibroblastic-shaped cells could be observed under microscope. Considering the survival rate and proliferation ability of BMSCs, Phenotypic analysis and differentiation experiments were carried out from passage 4 (Fig.1A). Next, we identified the surface marker molecules of BMSCs by immunofluorescence. Immunofluorescence analysis showed that BMSCs were positive expression of CD44, but negative expression of CD34 (Fig.1C). Additionally, BMSCs had the ability to differentiate into adipocytes and osteoblasts which showed by staining with Oil Red $O$ and Alizaran Red after 4 weeks of culturing in differentiation medium (Fig.1B). These results verifyed that BMSCs were successfully derivated.

\section{BMSCs homed to the lung tissues and ameliorated RILI}

In order to verify the success of BrdU labeling, we used immunofluorescence technology to verify BrdU Labeled BMSCs. The results showed that BrdU Labeled BMSCs produced bright green fluorescence, which proved that BrdU-Labeled BMSCs successfully. Subsequently, BrdU-labeled BMSCs were injected 
into mice through tail vein with irradiation. Immunofluorescence technique was used to monitor BMSCs BrdU-labeling. BrdU-labeled BMSCs with irradiation group produced stronger green fluorescence signal after 4 weeks (Fig.2A). To evaluate the therapeutic effects of BMSCs on RILI, we observed pathological changes in the lung tissues. Compared with the Control group, irradiation led to severe pathological changes in the lung tissues, including structural destruction, vasodilation and congestion, and infiltration of inflammatory cells. Pathological changes in the BMSCs group significantly alleviated. Similarly, we determined the protein expression levels of TNF-aand IL-6 in lung tissues, compared with the control groups, expression levels of TNF- $\alpha$ and IL- 6 were dramatically increased under irradiated stimulation, suggesting radiation causes a profound inflammatory response, in which markedly reduces in brdulabeled BMSCs with irradiation group (Fig.2B).

\section{BMSCs differentiates into alveolar epithelial cells}

In order to verify the changes of BMSCs after homing, immunofluorescence was used to assess the differentiation of BMSCs. Compared with the control group, the protein levels of AQP5 (a marker of alveolar type I cells) and of Pro-SPC (markers of alveolar type II cells) were significantly downregulated in the irradiation group. Compared with the irradiation group, the protein expression levels of AQP5, Pro-SPC were significantly upregulated in brdu-labeled BMSCs with irradiation group (Fig.3A, Fig.3B). The results explained that brdu-labeled BMSCs homed to the injured lung tissues and differentiated into alveolar cells, mainly alveolar type II cells .

\section{BMSCs regulate Angll/ACE2/Ang(1-7) axis}

It was hypothesized that the alleviating effect of RILI after BMSCs transplantation may be partly due to the increased expression of the ACE2 secreted AEC II which MSCs differentiate into. To verify the hypothesis, we first verify whether MSCs express ACE2 by immunofluorescence and western blotting. We selected ACE2 positive samples as control, the results found that MSCs did not express ACE2 protein (Fig.4A). Next to futher investigate whether BMSCs exert a critical effect on regulating Angll/ACE2/Ang(17) axis, western blotting was used to detect related proteins expression of Angll/ACE2/Ang(1-7) axis. Compared with the control group, the protein levels of Angll, ACE and AT1R were significantly upregulated (Fig.4B,4D,4E), but the protein levels of ACE2 and Ang(1-7) and the mRNA levels of MasR were remarkably downregulated in the irradiation group, which were reversed by BMSCs treatment (Fig.4C,4F,4G). Compared with the irradiation group, the protein levels of Angll, ACE and AT1R were significantly downregulated but the protein levels of ACE2 and Ang(1-7) and the mRNA levels of MasR were remarkably upregulated in the brdu-labeled BMSCs with irradiation group. As expected, these results suggested that BMSCs could regulate Angll/ACE2/Ang(1-7) axis to ameliorated RILI.

\section{BMSCs suppressed NF-KB/MAPK pathways under in vitro}


To further investigate whether the MAPK signaling pathway was associated with protective mechanism of BMSCs, Western blotting analysis was used to examined the MAPK activation level in lung tissue. Compared with the control group, irradiation substantially induced the phosphorylation of p38, ERK1/2 and Jnk, but p38, ERK1/2 and Jnk activation was blocked by BMSCs treatment (Fig.5A,5B,5C). Furthermore, we found that NF-кB was also activated rapidly under irradiation. However, the phosphorylation of NF-KB p65 in irradiated mice was dramatically inhibited by BMSCs treatment (Fig.5D). These results suggested that BMSCs may block the activation of NF-KB/MAPK signaling in RILI in mice.

\section{Discussion}

Nowadays, radiotherapy has become one of the most important treatment methods for thoracic tumors[17]. Although advanced radiation techniques can decrease radiation-related toxicity and increase the survival rate, it is still inevitable for occurrence of RILI, which limits the maximum dose for thoracic radiotherapy and reduces tumor control efficiency. RILI occurs in about $5-20 \%$ of patients with thoracic tumor clinically [2]. It is mainly divided into two stages: early radiation pneumonia and late radiation pulmonary fibrosis. Currently, molecular events the development of RILI is not fully elucidated [18]. Furthermore, there are no effective trearments for improving the clinical outcome of RILI [19]. BMSCs, a new treatment, have mediated well many beneficial therapeutic effects in various diseases [3,20]. Hence, we speculated whether the BMSCs can alleviate RILI. In this study, we demonstrated a possible mechanism of BMSCs in the treatment of RILI.

In this study, we successfully established BMSCs from rat thighbone and invetigated phenotype and differentiation potential of BMSCs. To verify whether BMSCs can alleviate the pathological changes of RILI, HE and immunohistochemistry techology were used to detect the pathological changes of lung tissue and the changes of inflammatory factors. In mice model, the irradiated lung tissues induced severe lung injury, including blood capillary congestion and dilatation, destruction of alveolar histological structure and inflammatory cells infiltration. It is reported that RILI was associated with expressed highly proinflammatory mediators, including IL-6 and TNF-a [21]. Our analysis showed the obviously elevated levels of IL-6 and TNF-a under irradiation. This result is consistent with the previous results [22]. However, After infusion of BMSCs, the injured lung tissues showed more remarkable relief with the relative reduction of alveolar tissue damage and lower degree of inflammation. Furthermore, BMSCs also reduced the expression levels of systemic TNF-a and IL-6. These results suggested that the protective effects of BMSCs were associated with anti-inflammatory, which consistented with previous studies [23]. In addition, previous research had reported the possibility of differentiation of BMSCs into alveolar epithelial cells $[24,25]$. The differentiation ability of BMSCs can help repair the integrity of alveolar epithelium cells, reducing RILI. In our study, we found that BrdU-labeled BMSCs can home to the injured lung tissues. Compared with irradiation group, the expression of Pro-spc (a marker of alveolar type II cells) and AQP5 (markers of alveolar type I cells) was markedly upregulated injected with BMSCs exposed to irradiation. Indeed, BMSCs mainly differentiated into alveolar type II cells. These results suggested that the BMSCs 
may home injured lung tissues and differentiate into alveolar cell especially alveolar type II cells to prevent RILI.

The renin-angiotensin system (RAS) plays a significant role in modulating blood pressure homeostasis, as well as fluid and salt balance[26]. Whereas, there is a growing body of evidence suggested that it was also important in regulating inflammatory responses. These effects are considered to be regulated mainly via the Angll/ACE2/Ang(1-7) axis [27]. Ang II plays as a critical regulatory molecule in the RAS system and promotes inflammatory process by increasing the release of inflammatory cytokines combined with the AT1R [28]. ACE2, a homologue of ACE and a negative regulator of RAS, is expressed in numerous tissues especially the lung alveolar epithelial cells, kidney, heart and plays a key role in regulating inflammatory responses[5]. Recent studies have shown that ACE2 significantly inhibits inflammatory responses [29]. However, nothing is known about Angll/ACE2/Ang(1-7) function in RILI. Our research found that the protein levels of Angll, ACE and AT1R were markedly upregulation in lung tissues under irradiation compared with control group, but irradiation resulted in significant downregulation of ACE2, Ang (1-7) and MasR protein. However, After infusion of BMSCs, the effects reversed these results which protein expression levels of AT1R, Ang II and ACE were significant decrease, whereas protein expression levels of ACE2, Ang(1-7) and MasR was dramatically increased. Besides, We verified that BMSCs do not express ACE2 by immunohistochemistry and western blotting. These results supported the hypothesis that the AEC II generation following injection of MSCs may responsible for the alleviation of RILI, at least in part due regulating Angll/ACE2/Ang(1-7) axis.

However, in order to explore how ACE2 plays an anti-inflammatory role, we tested the classic antiinflammatory pathway NF-KB/MAPK signaling pathway, which plays an important role in immune and inflammatory responses [30]. Previous studies have reported that NF-KB/MAPK signaling pathway was activated in inflammatory diseases and inhibiting it can effectively reduce the disease [31]. Thus, we investigated whether the NF-KB/MAPK signaling pathway was associated with the development of RILI by western blots. The results found that treatment with BMSCs under irradiation significantly inhibited the activition of p38, ERK, Jnk and NF-KB compared with Irradiation group. These results suggested that NF$\mathrm{KB} / \mathrm{MAPK}$ signaling pathway might be involved in the protective mechanism of BMSCs in RILI.

\section{Conclusions}

In conclusion, we have demonstrated treatment with BMSCs can alleviate RILI. Our study have revealed that the protective mechanism of BMSCs is associated with upregulating the ACE2/Ang (1-7) axis and inhibiting the NF-KB/MAPK signaling pathway, which will provide experimental basis for better treatment of RILI in the future.

\section{Abbreviations}

RILI: Radiation-induced lung injury; 
MSCs: Mesenchymal stem cells;

RT: Radiation therapy

RP: Radiation pneumonitis;

RILF: Radiation-induced lung fibrosis;

ALI: Acute lung injury ;

ARDS: Acute respiratory distress syndrome;

RAS: renin-angiotensin system;

Ang-(1-7): angiotensin-(1-7);

MAPKs: Mitogen-activated protein kinases;

IL-6: Interleukin6;

TNF-a: Tumor necrosis factor a;

DAB: 3,3-diaminobenzidin;

\section{Declarations}

\section{Funding}

This review was supported by the following funds: National Natural Science Foundation of China (81403150), Natural Science Foundation of Shandong Province of China (ZR2020MH389, ZR2020QH208), Shandong Provincial Medical and Health Science and Technology Development Project (2019WS187), Developmental Project of Traditional Chinese Medical Sciences and Technology of Shandong Province (2015-325), Innovation Project of Shandong Academy of Medical Sciences (201856), Key R \& D project of Shandong Province (Major Key Technologies) (2016ZDJSO7A15), and Academic Promotion Programme of Shandong First Medical University (2019QL007).

\section{Acknowledgements}

We would like to thank the National Natural Science Foundation of China for their support of this study.

\section{Authors'contributions}

YYZ and SML designed and conducted the study and performed animal study. NSY, 
and CCS performed the experiments. NSY analyzed the data and wrote the paper. All authors read and approved the final manuscript.

\section{Availability of data and materials}

Not applicable.

\section{Ethical approval and consent to participate}

This study was approved by the Ethics Committee of The First Affiliated Hospital of Shandong First Medical University. Our institution's committee on human research gave approval for this study, and all participants gave informed consent.

All animal experiments were approved by the Institutional Animal Care and Use Committee of The First Affiliated Hospital of Shandong First Medical University. Treatment of experimental animals followed the internationally recognized $3 \mathrm{R}$ principle, that is, replacement, reduction, and refinement of experimental animals and the welfare ethics of experimental animals in accordance with the requirements of the guidelines for ethical review of experimental animal welfare (GB/T 35892-2018) of China.

\section{Consent for publication}

Not applicable.

\section{Competing interests}

The authors declare that there is no conflict of interests.

\section{References}

1. Xu T. Zhang Y, Chang P. Gong S, Shao L. Dong L. Mesenchymal stem cell-based therapy for radiationinduced lung injury. Stem Cell Res Ther. 2018;9:18.

2. Giuranno L. lent J, De Ruysscher D, Vooijs MA. Radiation-induced lung injury (RILI). Front Oncol. 2019;9:877.

3. Gu W. Hong X, Potter C. Qu A, Xu Q. Mesenchymal stem cells and vascular regeneration. Microcirculation. 2017;24:e12324.

4. El-Hashim AZ. Khajah MA, Babyson RS. Renno WM, Ezeamuzie Cl. Benter IF, et al. Ang-(1-7)/ MAS1 receptor axis inhibits allergic airway inflammation via blockade of Src-mediated EGFR transactivation in a murine model of asthma. PLoS One. 2019;14:e0224163. 
5. Wiese O. Zemlin AE, Pillay TS. Molecules in pathogenesis: angiotensin converting enzyme 2 (ACE2). J Clin Pathol. 2021;74:285-90.

6. Chen Q. Liu J, Wang W. Liu S, Yang X. Chen M, et al. Sini decoction ameliorates sepsis-induced acute lung injury via regulating ACE2-Ang (1-7)-Mas axis and inhibiting the MAPK signaling pathway. Biomed Pharmacother. 2019;115:108971.

7. Han S. Gu F, Lin G. Sun X, Wang Y. Wang Z, et al. Analysis of clinical and dosimetric factors influencing radiation-induced lung injury in patients with lung cancer. J Cancer 2015;11:1172-8.

8. Huang Y. Zhang W, Yu F. Gao F. The Cellular and molecular mechanism of radiation-induced lung injury. Med Sci Monit. 2017;23:3446-50.

9. Tao X. Sun M, Chen M. Ying R, Su W. Zhang J, et al. HMGB1-modified mesenchymal stem cells attenuate radiation-induced vascular injury possibly via their high motility and facilitation of endothelial differentiation. Stem Cell Res Ther. 2019;10:92.

10. Ulivi V. Tasso R, Cancedda R. Descalzi F. Mesenchymal stem cell paracrine activity is modulated by platelet lysate: induction of an inflammatory response and secretion of factors maintaining macrophages in a proinflammatory phenotype. Stem Cells Dev 2014;16:1858-69.

11. Li XC. Zhu D, Zheng X. Zhang J, Zhuo JL. Intratubular and intracellular renin-angiotensin system in the kidney: a unifying perspective in blood pressure control. Clin. Sci. (Lond., Engl:: 1979) 2018;132:1383-1401..

12. Santos RAS. Sampaio WO, Alzamora AC. Motta-Santos D, Alenina N. Bader M, et al. The ACE2/Angiotensin-(1-7)/MAS Axis of the renin-angiotensin system: focus on angiotensin-(1-7). Physiol Rev. 2018;98:505-53.

13. Imai Y. Kuba K, Rao S. Huan Y, Guo F. Guan B, et al. Angiotensin-converting enzyme 2 protects from severe acute lung failure. Nature. 2005;436:112-6.

14. Zhang D. Li X, Hu Y. Jiang H, Wu Y. Ding Y, et al. Tabersonine attenuates lipopolysaccharide-induced acute lung injury via suppressing TRAF6 ubiquitination, Biochem. Pharmacol 2018;154:183-92.

15. Kim SR. Kim HJ, Kim DI. Lee KB, Park HJ. Jeong JS, et al. Blockade of interplay between IL-17A and endoplasmic reticulum stress attenuates LPS-Induced lung injury, Theranostics 2015;5:1343-62.

16. Lee JH. Min DS, Lee CW. Song KH, Kim YS. Kim HP. Ginsenosides from Korean Red Ginseng ameliorate lung inflammatory responses: inhibition of the MAPKs/NF-kappaB/c-Fos pathways, $\mathrm{J}$ Ginseng Res. 2018;42: 476-84.

17. Hanania AN. Mainwaring W, Ghebre YT. Hanania NA, Ludwig M. Radiation-induced lung injury: assessment and management. Chest. 2019;156:150-62.

18. Zanoni M. Cortesi M, Zamagni A. Tesei A. The role of mesenchymal stem cells in radiation-induced lung fibrosis. Int J Mol Sci. 2019;20:3876.

19. Zhang K. Yang S, Zhu Y. Mo A, Zhang D. Liu L. Protection against acute radiation-induced lung injury: a novel role for the anti-angiogenic agent Endostar. Mol Med Rep 2012;2:309-15. 
20. Mishra VK. Shih HH, Parveen F. Lenzen D, Ito E. Chan TF, et al. Identifying the therapeutic significance of mesenchymal stem cells. Cells. 2020;9:1145.

21. Beach TA. Groves AM, Williams JP. Finkelstein JN. Modeling radiation-induced lung injury: lessons learned from whole thorax irradiation. Int J Radiat Biol. 2020;96:129-44.

22. Zheng L. Zhu Q, Xu C. Li M, Li H. Yi PQ, et al. Glycyrrhizin mitigates radiation-induced acute lung injury by inhibiting the HMGB1/TLR4 signalling pathway. J Cell Mol Med. 2020;24:214-26.

23. Hao Y. Ran Y, Lu B. Li J, Zhang J. Feng C, et al. Therapeutic effects of human umbilical cord-derived mesenchymal stem cells on canine radiation-induced lung injury. Int J Radiat Oncol Biol Phys. 2018;102:407-16.

24. Liang Y. Yin C, Lu XI. Jiang H, Jin F. Bone marrow mesenchymal stem cells protect lungs from smoke inhalation injury by differentiating into alveolar epithelial cells via Notch signaling. J Biosci. 2019;44:2.

25. Li Q. Chen X, Li J. Marrow-derived mesenchymal stem cells regulate the inflammatory response and repair alveolar type II epithelial cells in acute lung injury of rats. $J$ Int Med Res. 2020;48:300060520909027.

26. Simões e Silva AC. Silveira KD, Ferreira AJ. Teixeira MM. ACE2, angiotensin-(1-7) and Mas receptor axis in inflammation and fibrosis. Br J Pharmacol. 2013;169:477-92.

27. Tan WSD. Liao W, Zhou S. Mei D, Wong WF. Targeting the renin-angiotensin system as novel therapeutic strategy for pulmonary diseases. Curr Opin Pharmacol. 2017; 40:9-17.

28. Wang X. Ye Y, Gong H. Wu J, Yuan J. Wang S, et al. The effects of different angiotensin II type 1 receptor blockers on the regulation of the ACE-Angll-AT1 and ACE2-Ang(1-7)-Mas axes in pressure overload-induced cardiac remodeling in male mice. J Mol Cell Cardiol. 2016;97:180-90.

29. Rodrigues Prestes TR. Rocha NP, Miranda AS. Teixeira AL, Simoes-E-Silva AC. The Anti-inflammatory potential of ACE2/Angiotensin-(1-7)/Mas receptor axis: evidence from basic and clinical research. Curr Drug Targets. 2017;18:1301-13.

30. Li C. Zhao B, Lin C. Gong Z, An X. TREM2 inhibits inflammatory responses in mouse microglia by suppressing the PI3K/NF-KB signaling. Cell Biol Int. 2019;43:360-72.

31. Meng Y. Yu CH, Li W. Li T, Luo W. Huang S, et al. Angiotensin-Converting Enzyme 2/Angiotensin-(17)/Mas axis protects against lung fibrosis by inhibiting the MAPK/NF-KB pathway, Am J Respir Cell Mol Biol. 2014;50: 723-36.

\section{Figures}


A

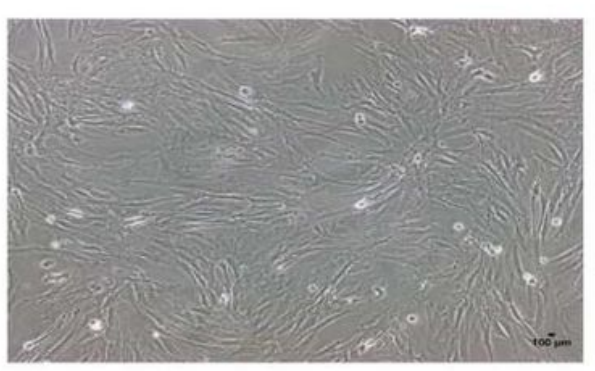

CD34

C

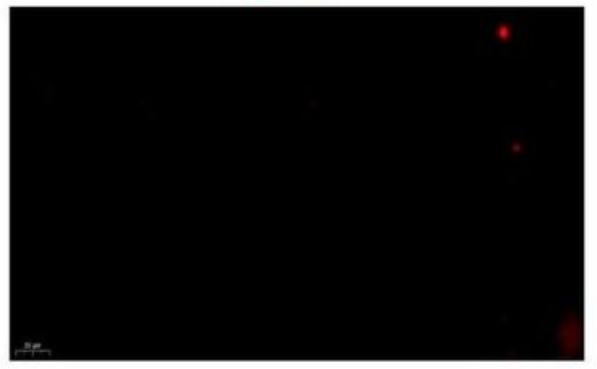

CD44

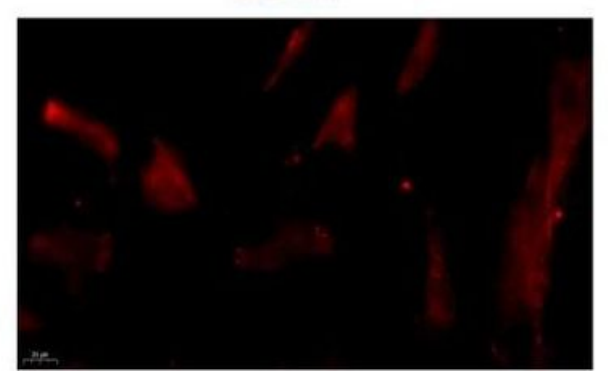

B

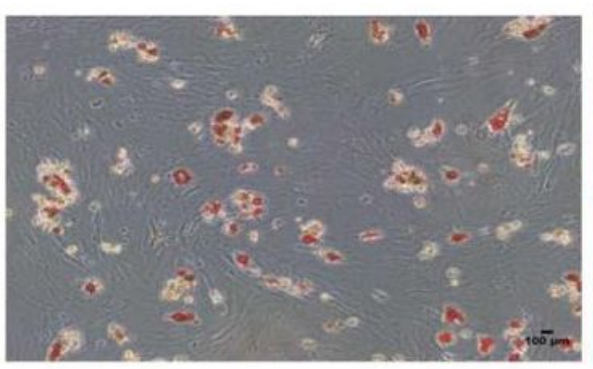

DAPI

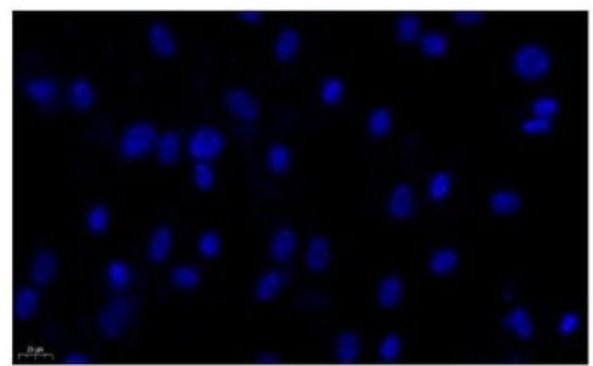

DAPI

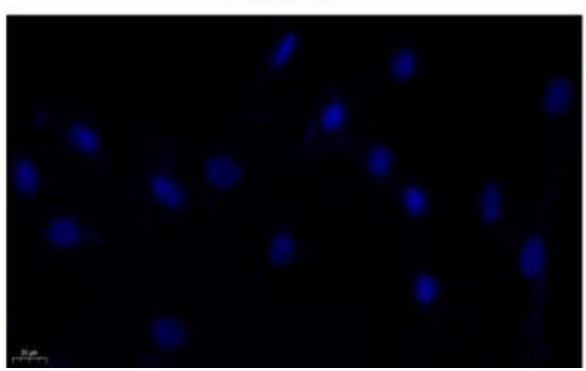

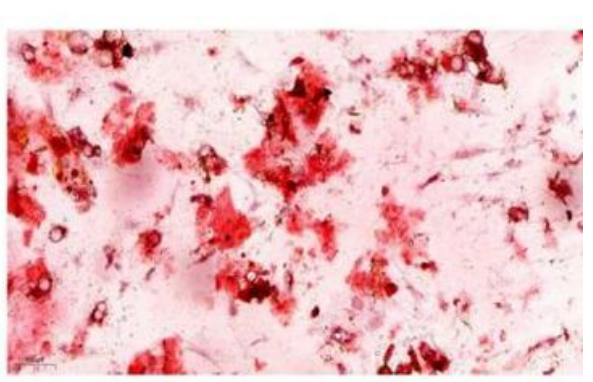

Merge

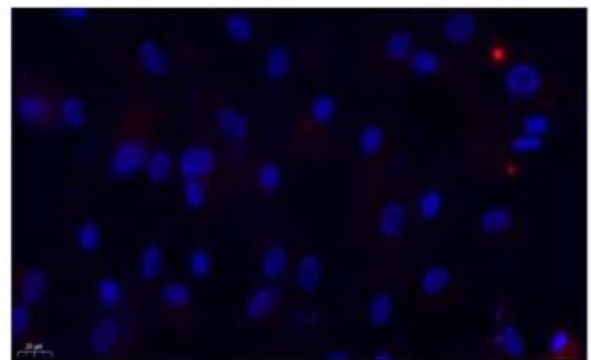

Merge

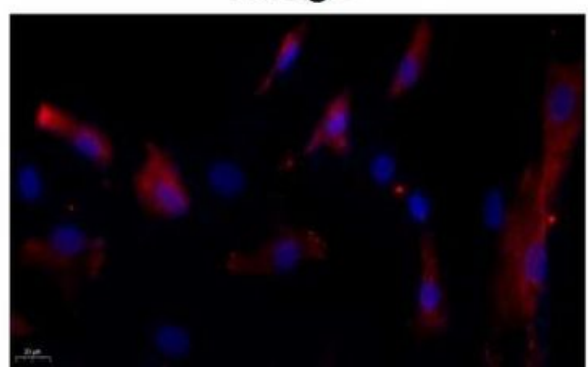

\section{Figure 1}

Establishment and identification of BMSCs. A BMSCs culture the third generation of cells, Scale bars, 100 $\mu \mathrm{m}$. B Identification of osteogenic and adipogenic cells by Alizarin red staining and oil red $O$ staining. Scale bars, $100 \mu \mathrm{m}$. C Surface molecular expression of the BMSCs, which were negative in CD34 expression, but positive in CD44 expression. Scale bars, $20 \mu \mathrm{m}$. BMSCs: Bone mesenchymal stem cells 
A

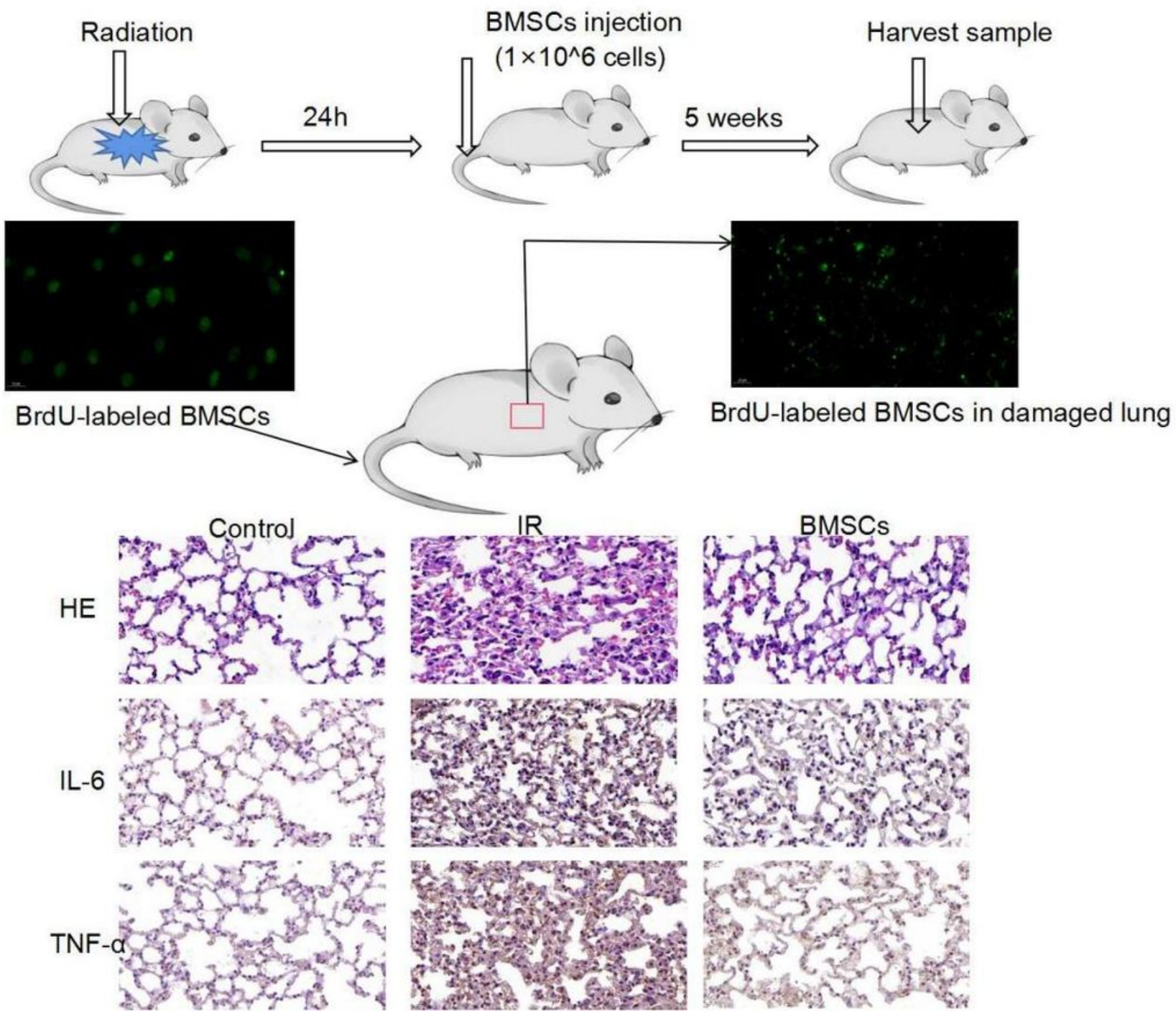

Figure 2

BMSCs homed to the lung tissues and histopathological changes in lung tissues. A Identification of BrdULabeled BMSCs in vitro and vivo, which showed strong green fluorescent staining in BrdU expression. Scale bars, $20 \mu \mathrm{m}$. B Histopathological changes in lung tissues and the expression changes of IL-6 and TNF-a from each experimental group by H\&E staining and immunohistochemical staining. Scale bars, $20 \mu \mathrm{m}$. IL-6: Interleukin6; TNF-a: Tumor necrosis factor a. 


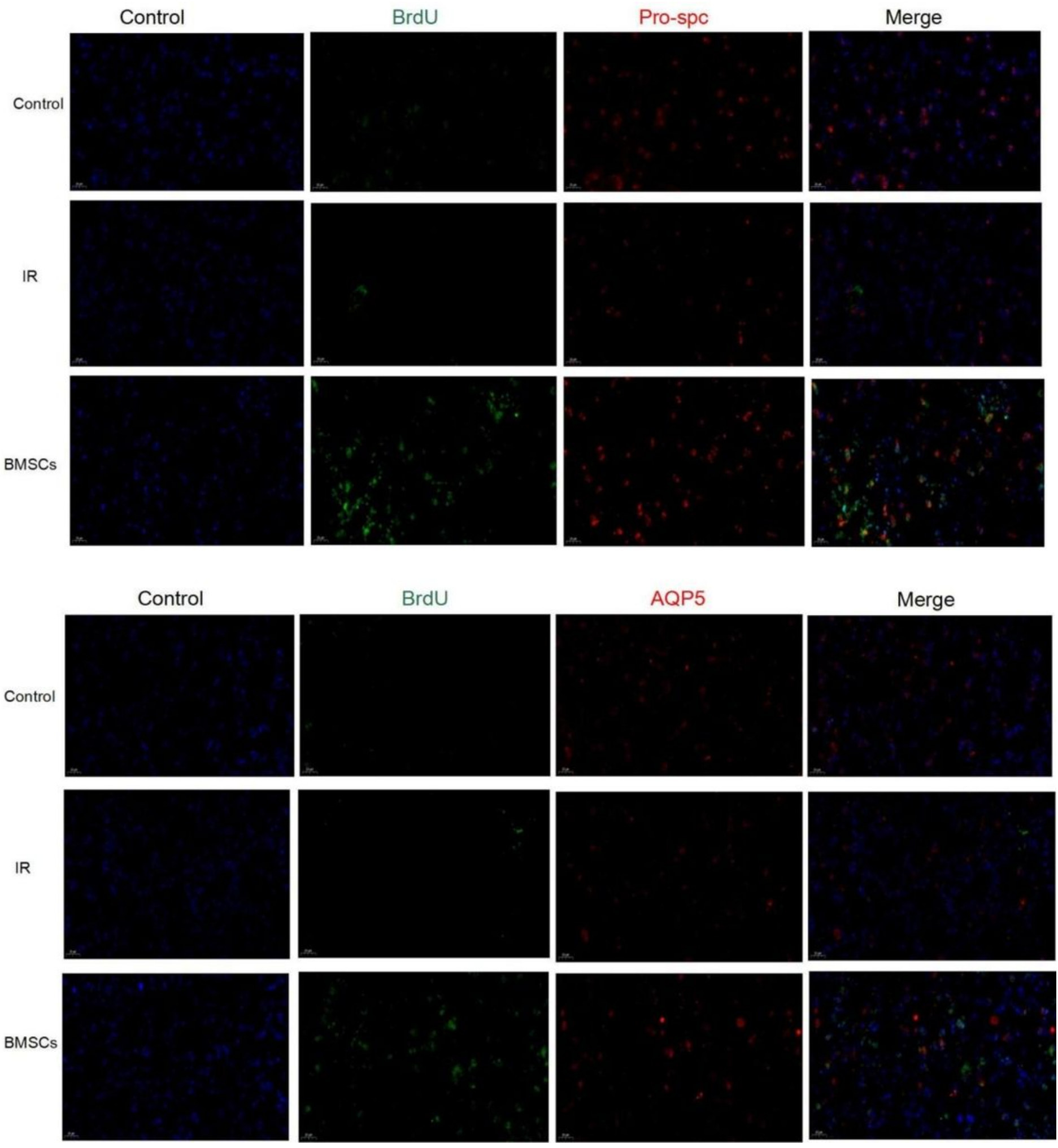

\section{Figure 3}

BMSCs differentiated into alveolar epithelial cells in lung tissues of RILI. The expression levels of AQP5 and Pro-spc in the lung tissues were measured by immunofluorescence. Scale bars, $20 \mu \mathrm{m}$. 


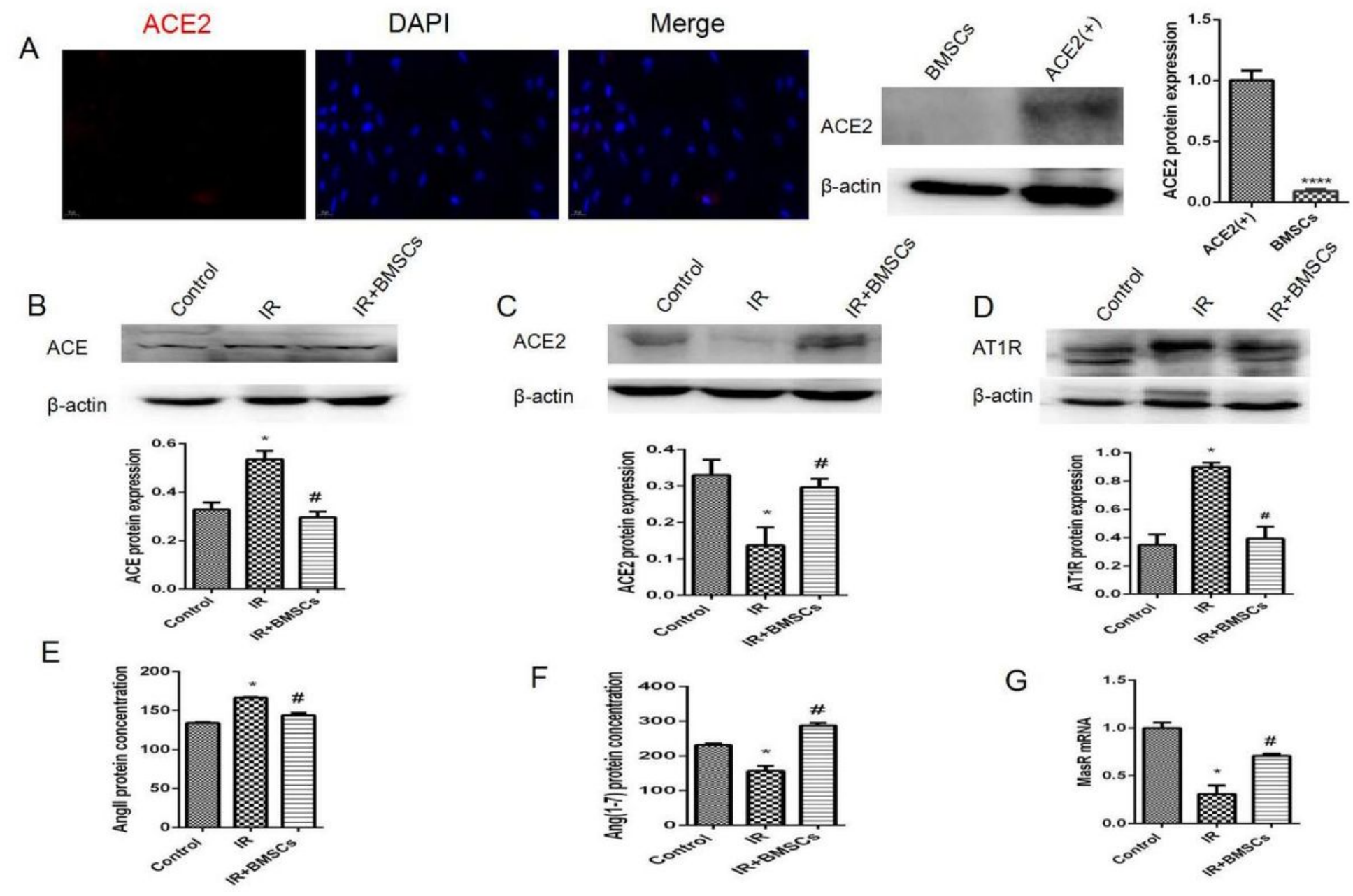

\section{Figure 4}

The related protein expression of angll/ACE2/ang(1-7) axis. B The protein expression levels of ACE in the lung tissues were measured by western blot assay and quantification of ACE protein expression. $C$ The protein expression levels of ACE2 in the lung tissues were measured by western blot assay and quantification of ACE2 protein expression. D The protein expression levels of AT1R in the lung tissues were measured by western blot assay amd quantification of AT1R protein expression. E The protein expression levels of Angll in the lung tissues were measured by ELISA. $F$ The protein expression levels of Ang(1-7) in the lung tissues were measured by ELISA. G The mRNA expression levels of MasR in the lung tissues were measured by qRT-PCR. ${ }^{*}$ Compared with the contol group, $P<0.05$. \#Compared with the IR group, $\mathrm{P}<0.05,{ }^{\star \star \star *}$ Compared with the ACE2(+) group, $\mathrm{P}<0.0001$. 


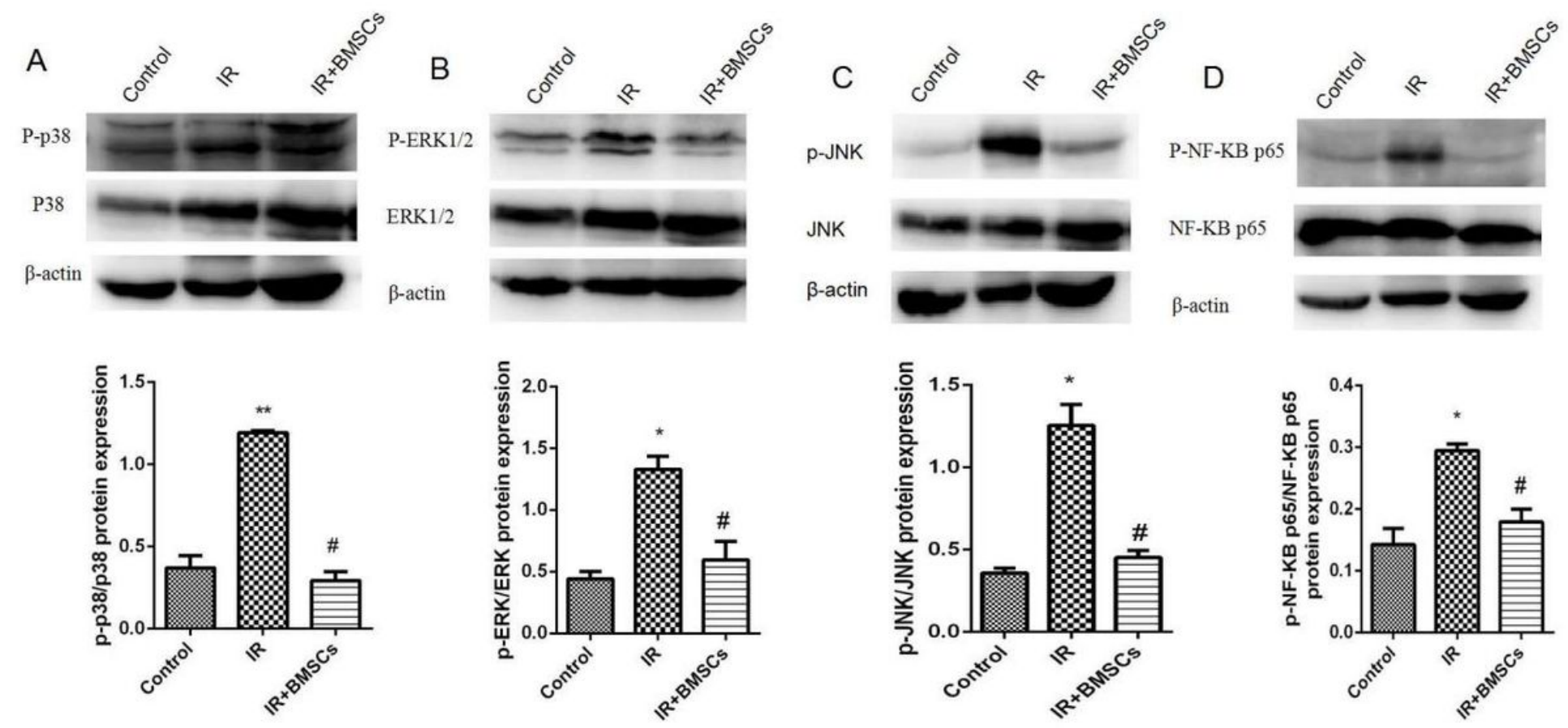

Figure 5

The expression level of NF-KB/MAPKs pathway protein in lung tissue. A The protein expression levels of P-P38 and P38 in the lung tissues were measured by western blot assay and quantification of P-P38/P38 protein expression. $B$ The protein expression levels of P-ERK1/2 and ERK $1 / 2$ in the lung tissues were measured by western blot assay and quantification of P-ERK1/2/ERK $1 / 2$ protein expression. $C$ The protein expression levels of P-JNK and JNK in the lung tissues were measured by western blot assay and quantification of P-JNk/JNK protein expression. D The protein expression levels of P-NF-KB P65 and NFKB P65 in the lung tissues were measured by western blot assay and quantification of P-NF-KB P65/NFKB P65 protein expression. *Compared with the contol group, $\mathrm{P}<0.05$. \#Compared with the IR group, $P<0.05$. 


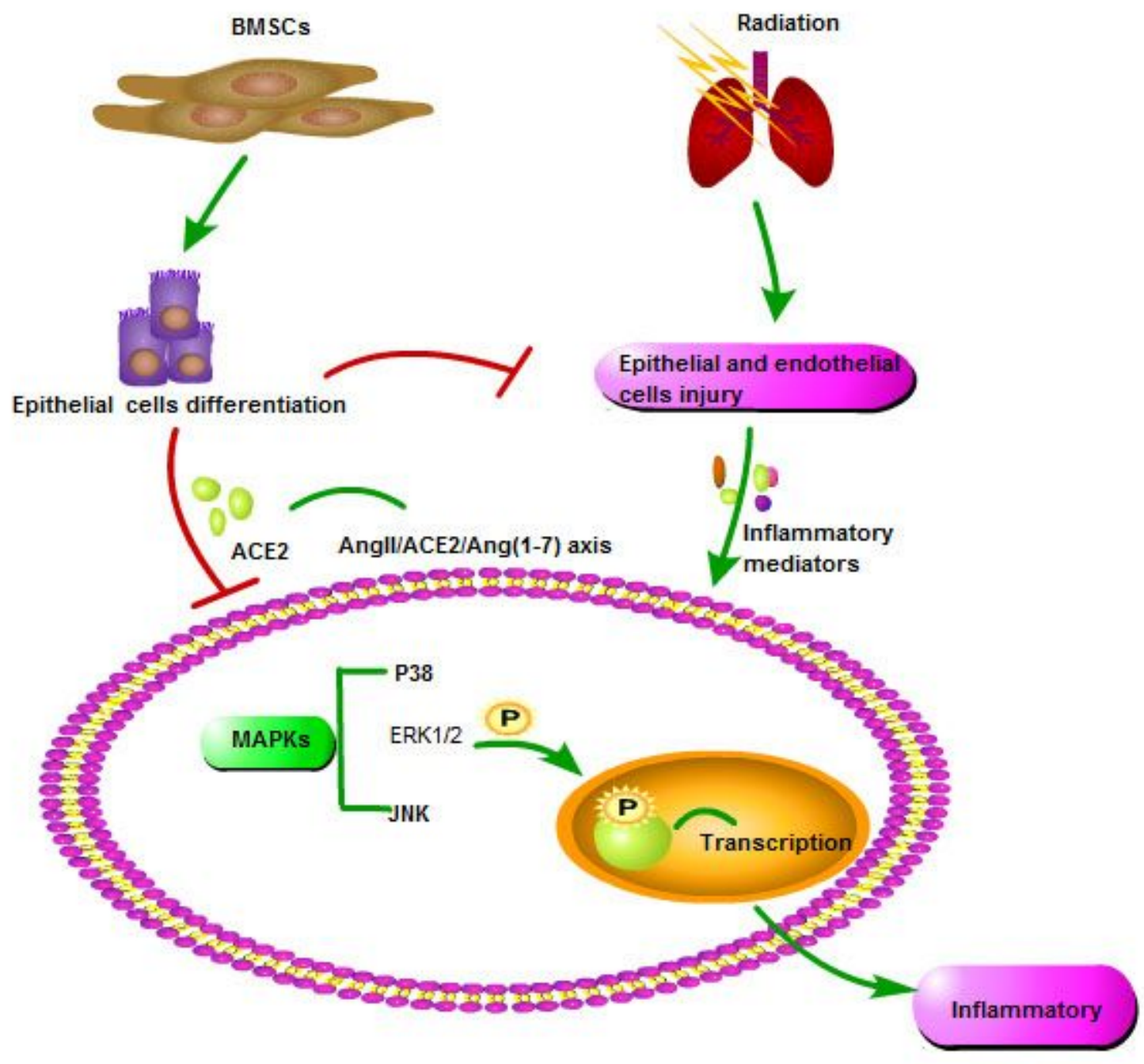

Figure 6

Illustration of possible mechanisms that bone marrow mesenchymal stem cells can not only repair some damaged epithelial cells but also produce ACE2 by differentiating into alveolar epithelial cells and inhibit MAPK pathway to alleviate radiation-induced lung injury. 